All-polymer solar cells based on photostable bis(perylene diimide) acceptor polymers

Peer-reviewed author version

LENAERTS, Ruben; CARDEYNAELS, Tom; Sudakov, Ivan; KESTERS, Jurgen; VERSTAPPEN, Pieter; MANCA, Jean; Champagne, Benoit; LUTSEN, Laurence; VANDERZANDE, Dirk; VANDEWAL, Koen; Goovaerts, Etienne \& MAES, Wouter (2019) All-polymer solar cells based on photostable bis(perylene diimide) acceptor polymers. In: SOLAR ENERGY MATERIALS AND SOLAR CELLS, 196, p. 178-184.

DOI: $10.1016 /$ j.solmat.2019.03.044

Handle: http://hdl.handle.net/1942/29029 


\section{All-Polymer Solar Cells Based on Photostable Bis(Perylene Diimide) Acceptor Polymers}

Ruben Lenaerts, ${ }^{1,2}$ Tom Cardeynaels, ${ }^{1,2,3}$ Ivan Sudakov, ${ }^{4}$ Jurgen Kesters, ${ }^{1,2}$ Pieter Verstappen, ${ }^{1,2}$ Jean Manca, ${ }^{5}$ Benoît Champagne, ${ }^{3}$ Laurence Lutsen, ${ }^{2}$ Dirk Vanderzande, ${ }^{1,2}$ Koen Vandewal, ${ }^{1,2}$ Etienne Goovaerts, ${ }^{4}$ Wouter Maes ${ }^{1,2}$

${ }^{1}$ UHasselt - Hasselt University, Institute for Materials Research (IMO-IMOMEC), Agoralaan 1, 3590 Diepenbeek, Belgium

${ }^{2}$ IMOMEC Division, IMEC, Wetenschapspark 1, 3590 Diepenbeek, Belgium

${ }^{3}$ University of Namur, Laboratory of Theoretical Chemistry, Theoretical and Structural Physical Chemistry Unit, Namur Institute of Structured Matter, Rue de Bruxelles 61, 5000 Namur, Belgium

${ }^{4}$ University of Antwerp, Department of Physics, Universiteitsplein 1, 2610 Antwerpen, Belgium

${ }^{5}$ UHasselt - Hasselt University, X-LAB, Agoralaan 1, 3590 Diepenbeek, Belgium 


\begin{abstract}
Fullerene-free organic photovoltaics have recently reached impressive power conversion efficiencies above $14 \%$ for single junctions, increasing their competitiveness with respect to alternative thin-film technologies. In most record devices, electron-donating conjugated polymers are combined with novel generation small molecule acceptors. All-polymer organic solar cells, on the other hand, still lag behind in efficiency, although they have specific advantages in terms of ink formulation and long-term operational stability. Another point of attention is the synthetic complexity of the active layer materials, notably on the side of the new acceptor molecules. Therefore, the present study focuses on the implementation of the stable and cost-effective perylene diimide structure as the key component of high-performance electron-accepting polymers. The synthesis, structural and optoelectronic characterization of four pushpull type copolymers containing the electron-deficient bis(perylene diimide) (bis-PDI) unit is reported, as well as the photovoltaic analysis of these acceptor materials in combination with a well-known donor polymer (PTB7-Th). The acceptor polymers differ in the electron-rich part of the alternating push-pull structure and their solar cell power conversion efficiencies range from 3.2 to $4.7 \%$. The maximum efficiency - the best performance achieved with a bis-PDI polymer so far - is obtained for the structurally most simple polymer, containing merely thiophene as the electron-rich building block. Controlled degradation under blue light in air is monitored by the bleaching of the relevant UV-Vis absorption bands, demonstrating high stability for the bis-PDI-thiophene containing polymers as compared to some prototype small molecule acceptors (FBR and ITIC).
\end{abstract}

\title{
Keywords
}

organic photovoltaics, fullerene-free, all-polymer, perylene diimide, synthetic complexity, photostability 


\section{Introduction}

Organic solar cells are a particular class of thin-film photovoltaics featuring attractive properties such as flexibility, semi-transparency and improved low-light performance. Moreover, the carbon-based lightharvesting materials do not pose problems with respect to toxicity or scarcity and the unprecedented high degree of freedom in the photoactive organic material design enables to optimize absorption, charge transport and energy level alignment.[1, 2] Traditionally, solution-processed bulk heterojunction organic photovoltaics (OPVs) consist of an electron-donating polymer or small molecule, blended together with a methanofullerene compound as electron acceptor. This approach has afforded power conversion efficiencies (PCEs) over 11\%.[3-6] Fullerenes, however, have rather high production costs with a high energy demand, do not contribute much to light harvesting and their use leads to stability issues and inherent energy losses.[7-11] As such, they can be considered a limiting factor for OPV efficiency, stability and cost. As a result, over the last 5 years, the interest of the PV community shifted to other thin-film technologies targeting similar applications (e.g. building-integrated photovoltaics), especially as this coincided with the explosive rise in efficiencies achieved with hybrid organic-inorganic perovskite solar cells. To address the obstacles listed above, but keeping the advantages of fully organic materials, alternative non-fullerene electron acceptors have been pursued. In initial studies, however, it has been very difficult to achieve efficiencies comparable to fullerene-based devices.[12] This situation has notably changed over the last years and PCEs over $14 \%$ have now been realized for single-junction OPV devices, overruling (by far) the best fullerene-based systems.[13-15] A record (certified) PCE of $17.3 \%$ has even been achieved for organic solution-processed tandem solar cells.[16] This impressive progress has created renewed enthusiasm in the field and new research questions related to the fundamental limitations of non-fullerene OPV have emerged.[17, 18]

Besides small molecules, electron accepting polymers are also possible alternatives to fullerenes. In terms of efficiency, all-polymer solar cells still lag behind, with the highest efficiencies around 11\%.[17, 19-21] However, there are no known fundamental causes for this lower performance. On the other hand, polymer-polymer combinations might have specific advantages in terms of flexibility, ink formulation as well as long-term thermal and mechanical stability.[22, 23]

From a structural point of view, the most successful small molecule non-fullerene acceptors (NFAs) to date, including ITIC and IEICO (see Supplementary data, Figure S28),[14] all have a similar backbone featuring strong intramolecular push-pull interactions. Another successful class of non-fullerene materials is based on fused aromatic diimides, notably naphthalene diimides (NDIs) and perylene diimides 
(PDIs). $[14,17]$ Although the efficiencies achieved with PDI acceptors are somewhat lower, these materials have important advantages in terms of synthetic complexity,[24] i.e. a low material synthesis cost, and stability. $[25,26]$ The key feature with respect to the molecular design of PDI-based acceptors has been to restrain their aggregation tendency and improve their miscibility with polymer donors. [27] In this respect, one of the simplest strategies is to link two PDIs at the so-called bay positions, with the resulting twist rendering the bis-PDI molecules non-planar. Jen et al. reached an OPV efficiency of 5.5\% using a pristine bis-PDI small molecule in combination with PTB7-Th as the electron donor polymer in an inverted solar cell stack.[28] A single push-pull conjugated polymer containing a bis-PDI 'pull' monomer and a dithiophene 'push' unit has been reported as well, affording a maximum PCE of 4.5\% (again with PTB7-Th as the donor).[29] As such, further progress can be envisaged when optimizing the molecular structure of bis-PDI acceptor polymers.

In this work, the synthesis as well as the structural and optoelectronic characterization of four low gap push-pull type copolymers containing the electron-deficient bis-PDI structure (Figure 1 ) is reported, as well as the analysis of the photovoltaic performance of these acceptor materials. Two of the prospected key advantages of bis-PDI acceptor polymers, their low synthetic complexity and high photostability, are investigated as well. We find that all four polymers exhibit high photostability and a maximum solar cell efficiency of $4.7 \%$ is obtained for PTbPDI, the structurally most simple polymer.
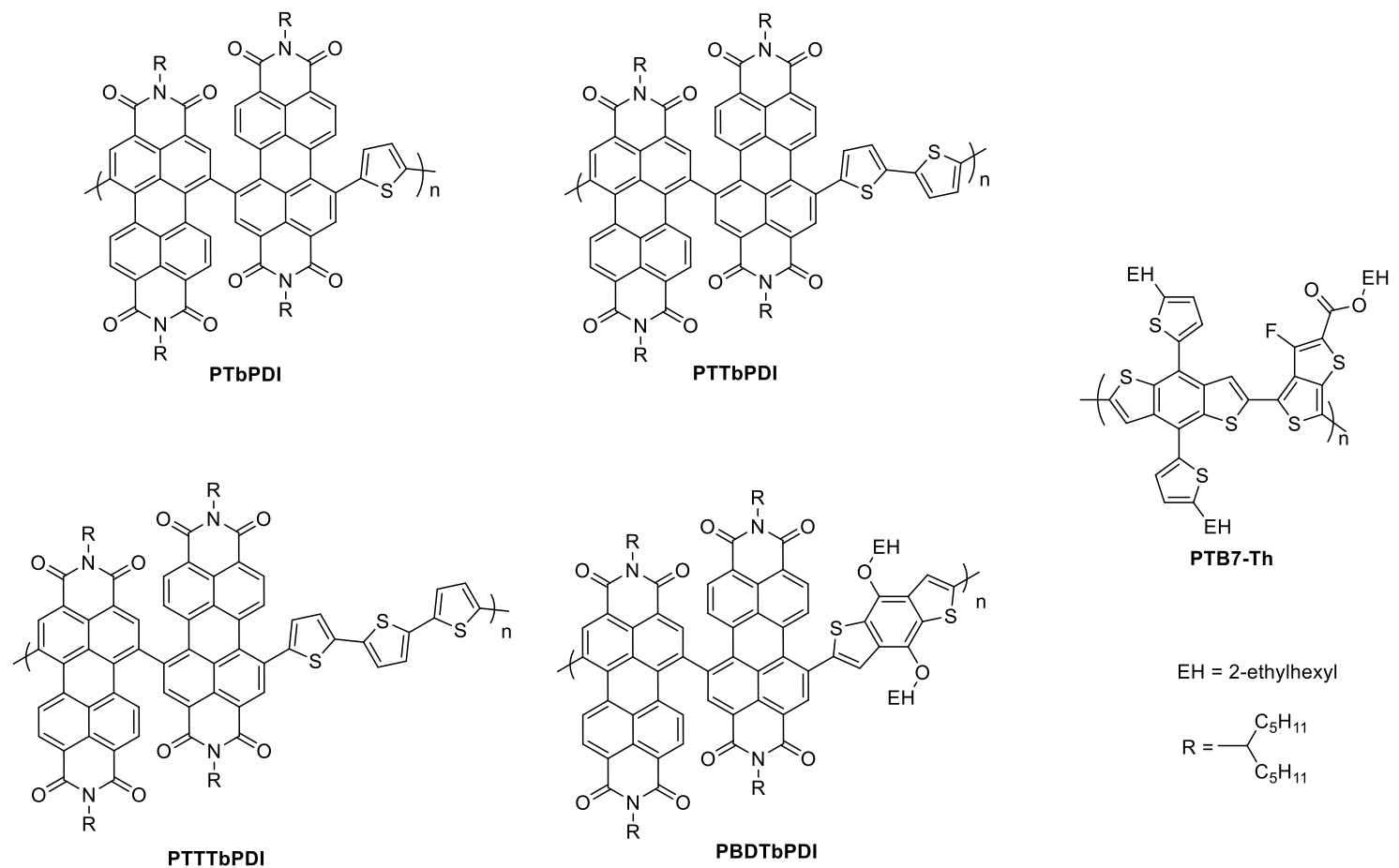

PTTTbPDI

Figure 1. Chemical structures of the bis-PDI acceptor polymers and the PTB7-Th donor polymer. 


\section{Results and discussion}

The four acceptor polymers synthesized and analyzed in this study differ in their electron-rich 'push' part, as the bis-PDI monomer is copolymerized with either thiophene (PTbPDI), dithiophene (PTTbPDI), [29] terthiophene (PTTTbPDI) or benzodithiophene (PBDTbPDI) (Figure 1).

The general synthetic route to prepare the acceptor polymers is depicted in Scheme 1. Monomers 1, [3033] 2, [34] 3, [35] 4 [35] and 5 [36-38] were synthesized according to slightly adapted literature procedures (see Supplementary data). The polymers PTbPDI, PTTbPDI, PTTTBPDI and PBDTbPDI were then prepared via Stille cross-coupling polycondensation of dibromo-bis-PDI 1 and the different donor units, i.e. 2,5bis(trimethylstannyl)thiophene (2), 5,5'-bis(trimethylstannyl)-2,2'-bithiophene (3), 5,5"bis(trimethylstannyl)-2,2':5',2'-terthiophene (4) and [4,8-bis(2-ethylhexyloxy)benzo[1,2-b:4,5$b^{\prime}$ ]dithiophene-2,6-diyl]bis(trimethylstannane) (5) (Scheme 1), followed by purification with sodium diethyldithiocarbamate as a palladium scavenger, Soxhlet extractions and precipitations. Gel permeation chromatography (GPC) analysis demonstrated number-average molar masses $\left(M_{\mathrm{n}}{ }^{\prime} \mathrm{s}\right)$ of $19,23,37$ and 30 $\mathrm{kg} \mathrm{mol}^{-1}$ for PTbPDI, PTTbPDI, PTTTbPDI and PBDTbPDI, respectively, all with a dispersity $(\theta)$ of 2.0 or lower (Table 1). All four polymers have similar low-lying lowest unoccupied molecular orbital (LUMO) levels around $-4.2 \mathrm{eV}$, as estimated from cyclic voltammetry (CV) experiments, while the highest occupied molecular orbital (HOMO) levels and HOMO-LUMO gaps $\left(E_{g}{ }^{E C}\right)$ differ depending on the electron-rich monomer used (Table 1). PTbPDI has the largest $E_{g}{ }^{E C}$ of the four polymers (1.81 eV), followed by PBDTbPDI (1.80 eV), PTTbPDI (1.73 eV) and PTTTbPDI (1.45 eV). The UV-Vis absorption spectra in solution (Figure 2, top) and film (Figure 5 and S26) show a gradual red-shift of the absorption onset, starting in film from 674 $\mathrm{nm}$ for PTbPDI to $742 \mathrm{~nm}$ for PTTbPDI, $761 \mathrm{~nm}$ for PBDTbPDI and $800 \mathrm{~nm}$ for PTTTbPDI (Table 1, Figure 5 and S26). A minor red-shift can be observed comparing the solution and film spectra, indicating additional stacking of the polymers in the solid state. The mass extinction coefficients in solution were also determined and are all in the same range, with maxima around $20-25 \mathrm{~L} \mathrm{~g}^{-1} \mathrm{~cm}^{-1}$ (Table 1, Figure 1). The low-energy intramolecular charge transfer absorption band of PTTTbPDI appears to be a bit less intense $\left(17 \mathrm{~L} \mathrm{~g}^{-1} \mathrm{~cm}^{-1}\right)$. Finally, the absorption spectra of the four acceptor polymers blended with PTB7-Th in a 1:1 weight ratio (Figure 2, bottom) show a very similar broad absorption profile with the main differences appearing below $550 \mathrm{~nm}$. 


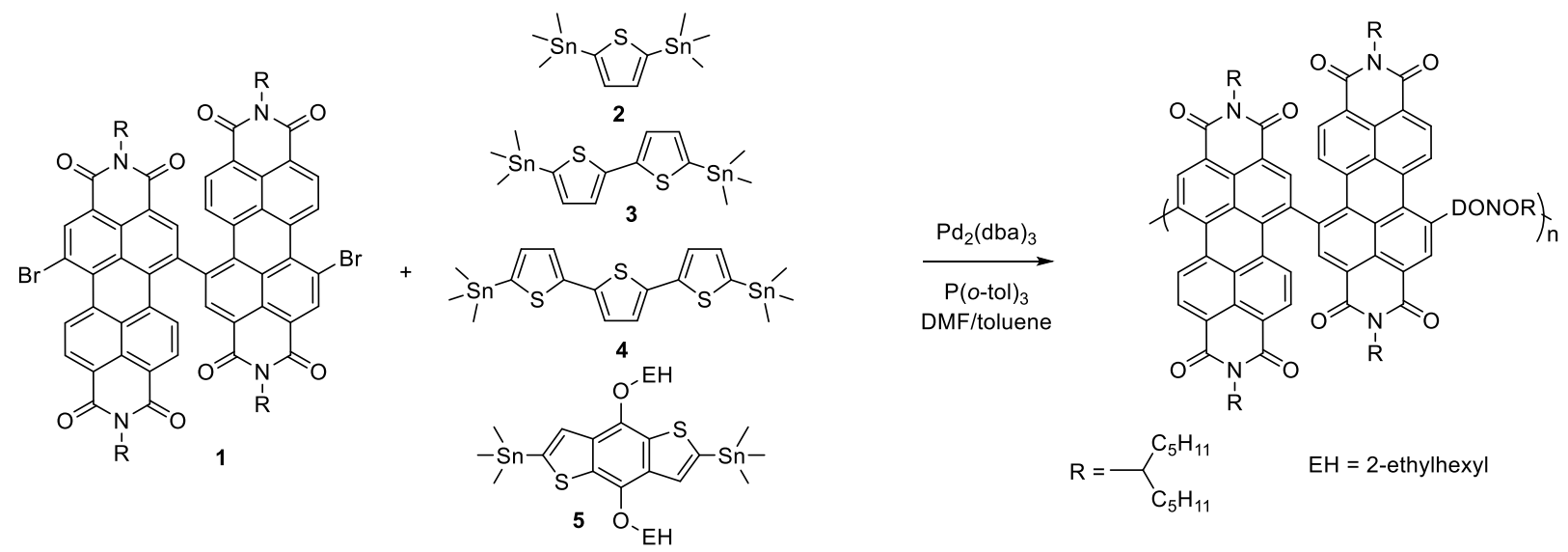

Scheme 1. Synthesis of polymers PTbPDI (1+2), PTTbPDI (1+3), PTTTbPDI (1+4) and PBDTbPDI (1+5) via Stille polycondensation.
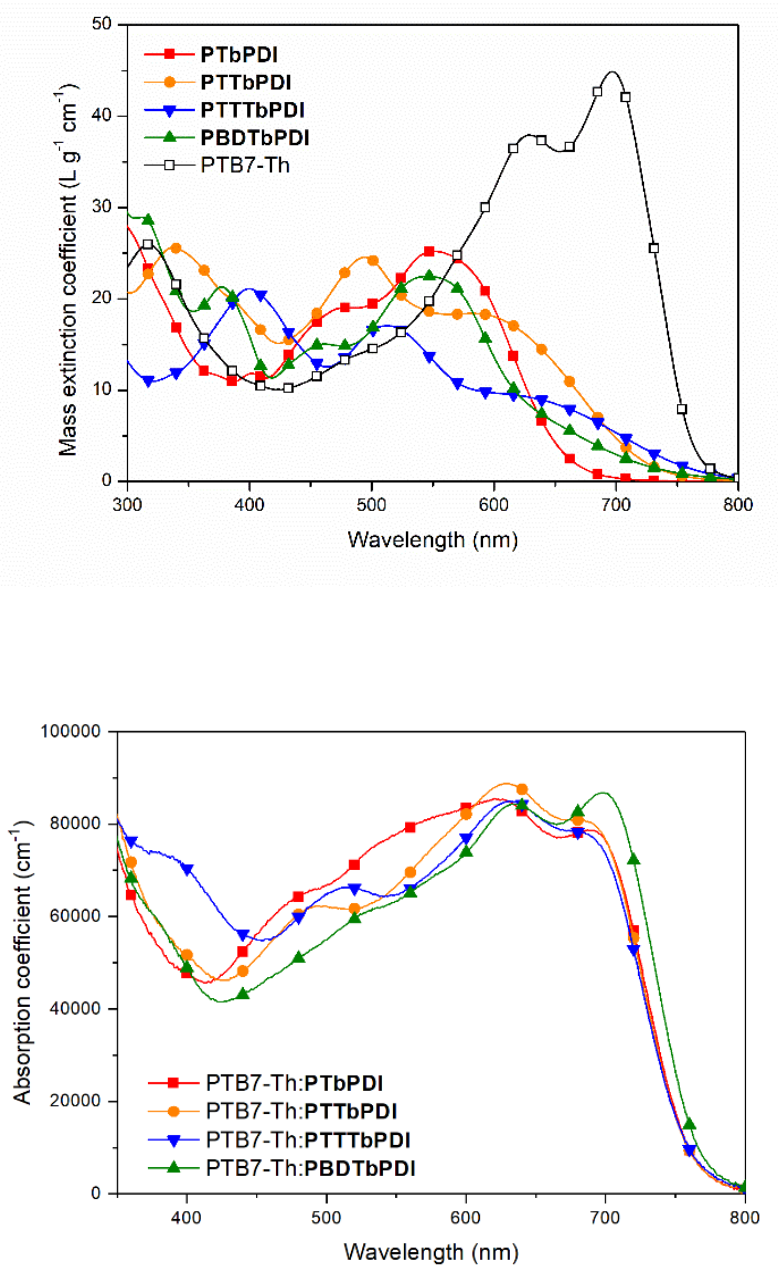

Figure 2. UV-Vis absorption spectra of the different polymers in chloroform solution (top) and thin-film spectra of the acceptor polymers blended with PTB7-Th in a 1:1 weight ratio (bottom). 
Table 1. Molar mass (distribution), optical and electrochemical properties of the donor and acceptor polymers.

\begin{tabular}{|c|c|c|c|c|c|c|c|}
\hline & $\begin{array}{c}M_{\mathrm{n}} \\
\left(\mathrm{kg} \mathrm{mol}^{-1}\right)\end{array}$ & D & $\begin{array}{c}\lambda_{\max } / \varepsilon \\
(\mathrm{nm}) /\left(\mathrm{L} \mathrm{g}^{-1} \mathrm{~cm}^{-1}\right)\end{array}$ & $\begin{array}{c}\mathrm{HOMO}^{a, b} \\
(\mathrm{eV})\end{array}$ & $\begin{array}{c}\text { LUMO }^{a, b} \\
(\mathrm{eV})\end{array}$ & $\begin{array}{l}E_{\mathrm{g}}^{\mathrm{ECC} c} \\
(\mathrm{eV})\end{array}$ & $\begin{array}{l}E_{\mathrm{g}} \mathrm{OPd} \\
(\mathrm{eV})\end{array}$ \\
\hline PTbPDI & 19 & 2.0 & $551 / 25$ & $-6.03(-6.36)$ & $-4.22(-3.81)$ & $1.81(2.55)$ & 1.84 \\
\hline PTTbPDI & 23 & 2.0 & $\begin{array}{l}494 / 25 \\
337 / 26\end{array}$ & $-5.96(-6.17)$ & $-4.23(-3.75)$ & $1.73(2.42)$ & 1.67 \\
\hline PTTTbPDI & 37 & 1.4 & $\begin{array}{l}512 / 17 \\
400 / 21\end{array}$ & $-5.68(-5.90)$ & $-4.23(-3.74)$ & $1.45(2.15)$ & 1.55 \\
\hline PBDTbPDI & 30 & 1.9 & $\begin{array}{l}545 / 23 \\
377 / 21\end{array}$ & $-6.05(-5.99)$ & $-4.25(-3.75)$ & $1.80(2.24)$ & 1.63 \\
\hline PTB7-Th & 25 & 6.0 & $697 / 45$ & -5.52 & -3.40 & 2.11 & 1.61 \\
\hline
\end{tabular}

${ }^{a}$ Determined by CV from the onset of oxidation/reduction. ${ }^{b}$ Calculated values (DFT) between brackets. ${ }^{c}$ Electrochemical gap. ${ }^{d}$ Optical gap, determined by the onset of the solid-state UV-Vis-NIR absorption spectrum.

From the CV measurements, a substantial increase in HOMO energy appears when moving from PTTbPDI to PTTTbPDI. To shed more light on this, density functional theory (DFT) calculations were carried out using Gaussian09[39] with the M06 exchange-correlation functional[40] and the 6-311G(d) basis set. For each of the acceptor polymers, two conformations of the same model oligomer were optimized to obtain the most stable form (Figure S20, S21). These conformers differ in the orientation of the two bis-PDI units with respect to each other. From the most stable conformers, it is clear that the HOMO energy increases linearly when going from PTbPDI to PTTBPDI and finally PTTTbPDI. When looking at the HOMO distribution in Figure S22, this becomes clear as the HOMO becomes less delocalized over the entire backbone and more localized on the donor unit. The energy of the LUMO, which is mostly localized on the bis-PDI units, remains almost constant throughout the entire series (Figure S22), as also concluded from the CV experiments. The calculated values for the HOMO-LUMO gap follow a linear trend, which is in line with the CV and UV-VIS data. The seemingly large jump in the CV data from PTTbPDI to PTTTbPDI is also observed in the calculations, but less in the UV-VIS data (Table 1). From the optimized geometries, it is clear that a large twist is present between the two PDI subunits, as well as between the PDI and the donor segments. This twisting of the polymer backbone hinders the stacking behavior of the acceptor polymers, allowing better mixing with the donor polymer.[27] 
Bulk heterojunction solar cell devices were then prepared with the different polymer acceptors to investigate their photovoltaic performance. PTB7-Th was chosen as the complementary absorbing low gap donor polymer $\left(M_{\mathrm{n}} 25 \mathrm{~kg} \mathrm{~mol}^{-1}\right)$.[41] Initially, OPV devices with a standard architecture (glass/ITO/PEDOT:PSS/active layer/Ca/Al) were fabricated and optimization studies were performed (Table S2-S5). As shown in Table 2 (and Figure 3a), the different material combinations gave optimal performances when processed from chloroform. Average PCEs ranging from 2.9 to $3.6 \%$ were obtained, with open-circuit voltages $\left(V_{o c}\right.$ 's), short-circuit current densities $\left(J_{s c}\right.$ 's) and fill factors (FFs) around 0.65-0.72 V, 7.75-10.97 mA cm${ }^{-2}$ and 0.43-0.53, respectively. With the standard architecture, PBDTbPDI turned out to be the best polymer in combination with PTB7-Th. When incorporated in OPV devices with an inverted architecture (glass/ITO/ZnO/active layer/MoO $/ \mathrm{Ag}$ ), the device parameters increased for all material combinations, with PCEs now ranging from 3.2 to $4.7 \%$ ( $V_{\text {oc }}$ 's from 0.71 to $0.73 \mathrm{~V}, \mathrm{~J}_{\mathrm{sc}}$ 's from 8.6 to $11.7 \mathrm{~mA} \mathrm{~cm}^{-2}$ and FFs from 0.52 to 0.56 ; Table 2, Figure $3 \mathrm{~b}$ ). With PTbPDI, a maximum PCE of almost $4.7 \%$ was reached, which, to our knowledge, is the highest reported efficiency for all-polymer solar cells based on bis-PDI polymers. The PTB7-Th:PTbPDI devices also show the highest external quantum efficiencies within the series, approaching $50 \%$ between 500 and $700 \mathrm{~nm}$ (Figure S23).

All polymers show about the same LUMO level (Table 1 ) and hence similar $V_{\text {oc }}$ 's are expected. The lower $V_{\text {oc }}$ values for the solar cells with a standard architecture in case of PTbPDI and PTTbPDI can likely be attributed to loss processes occurring at the contact between PEDOT:PSS and the active layer.[42] On the other hand, the PTB7-Th:PTTTbPDI and PTB7-Th:PBDTbPDI devices do not seem to suffer from substantial $V_{\text {oc }}$ losses related to the contacts. Also in the inverted devices, no such losses are apparent. As the $V_{\text {oc }}$ 's and FF's are about the same in the inverted devices, the PTB7-Th:PTbPDI blend performs best, since it affords the highest short-circuit current. The main difference among the three polymers with thiophene linkers is the gradual decrease in $J_{\mathrm{sc}}$ upon increasing the number of thiophene linkers. PTbPDI affords a $J_{\mathrm{sc}}$ that is $\sim 20 \%$ larger than the one for PTTbPDI, which in turn gives a higher $J_{s c}$ than PTTTbPDI. The same trend can be seen for both the standard and inverted devices. This could be related to the difference in absolute absorption coefficients, which slightly increase between 450 and $800 \mathrm{~nm}$ when the amount of thiophene units per chain decreases and the amount of bis-PDI units per chain increases (Figure 2), but the differences are not large enough to fully explain the variation in $J_{\mathrm{sc}}$. Another effect of adding more thiophene spacers between the bis-PDI units is the fact that this introduces a larger planar fraction within the polymer chains (see Figure S22), which counterbalances the effect of the bis-PDI moieties in restraining the aggregation tendency. 
Table 2. Solar cell parameters for optimized devices based on the bis-PDI acceptor polymers in combination with PTB7-Th.

\begin{tabular}{|c|c|c|c|c|c|}
\hline Donor:Acceptor ${ }^{a}$ & $\begin{array}{c}V_{o c^{b}} \\
\text { (V) }\end{array}$ & $\begin{array}{c}J_{S C^{b}} \\
\left(\mathrm{~mA} \mathrm{~cm}^{-2}\right)\end{array}$ & $\mathrm{FF}^{b}$ & $\begin{array}{l}\text { PCE }^{b} \\
(\%)\end{array}$ & $\begin{array}{c}\text { Best PCE } \\
(\%)\end{array}$ \\
\hline PTB7-Th:PTbPDI ${ }^{c}$ & 0.65 & $10.97 \pm 0.17$ & 0.43 & $3.04 \pm 0.07$ & 3.14 \\
\hline PTB7-Th:PTTbPDI ${ }^{c}$ & 0.68 & $8.75 \pm 0.19$ & 0.46 & $2.75 \pm 0.12$ & 2.81 \\
\hline PTB7-Th:PTTTbPDIc & 0.72 & $7.75 \pm 0.23$ & 0.53 & $2.96 \pm 0.10$ & 3.09 \\
\hline PTB7-Th:PBDTbPDI ${ }^{c}$ & 0.70 & $10.13 \pm 0.26$ & 0.50 & $3.56 \pm 0.04$ & 3.60 \\
\hline PTB7-Th:PTbPDI ${ }^{d}$ & 0.71 & $11.73 \pm 0.26$ & 0.54 & $4.51 \pm 0.09$ & 4.65 \\
\hline PTB7-Th:PTTPPDI ${ }^{d}$ & 0.72 & $9.80 \pm 0.22$ & 0.55 & $3.92 \pm 0.17$ & 4.03 \\
\hline PTB7-Th:PTTTbPDI ${ }^{d}$ & 0.73 & $8.60 \pm 0.30$ & 0.52 & $3.25 \pm 0.15$ & 3.39 \\
\hline PTB7-Th:PBDTbPDI $^{d}$ & 0.71 & $10.11 \pm 0.37$ & 0.56 & $4.07 \pm 0.12$ & 4.16 \\
\hline
\end{tabular}

a Total concentration of $12 \mathrm{mg} \mathrm{mL}^{-1}$ in chloroform, donor and acceptor in a 1:1 weight ratio. ${ }^{\mathrm{b}}$ Averages taken over 3 to 4 devices. c Standard device architecture: glass/ITO/PEDOT:PSS/active layer/Ca/Al. d Inverted device architecture: glass/ITO/ZnO/active layer/MoO $/ \mathrm{Ag}$.
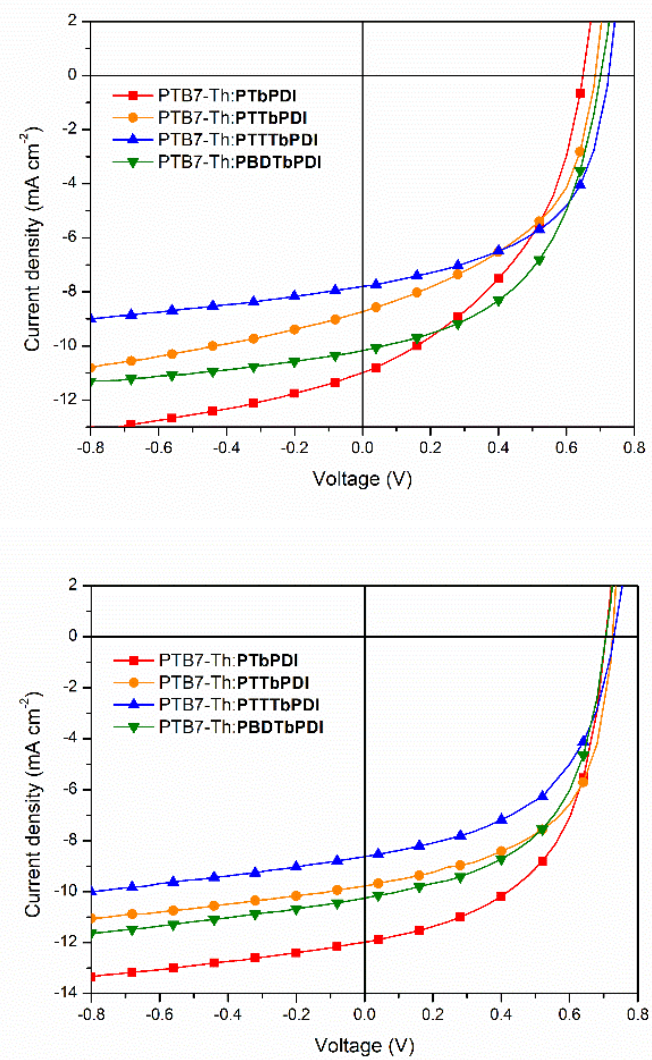

Figure 3. Current-density plots for the all-polymer solar cells (affording average PCEs) with standard (top) and inverted (bottom) architecture. 
From atomic force microscopy (AFM) analysis of the devices (Figure 4), no apparent phase separation could be observed, suggesting favorable blending of the donor and acceptor polymer components in all cases. Hole and electron (SCLC) mobilities extracted from hole and electron-only devices (see Supplementary data, Figure S24 and S25) are also very similar, in the range of $2-310^{-4} \mathrm{~cm}^{2} \mathrm{~V}^{-1} \mathrm{~s}^{-1}$ and $0.4-0.710^{-4} \mathrm{~cm}^{2} \mathrm{~V}^{-1} \mathrm{~s}^{-1}$, respectively.
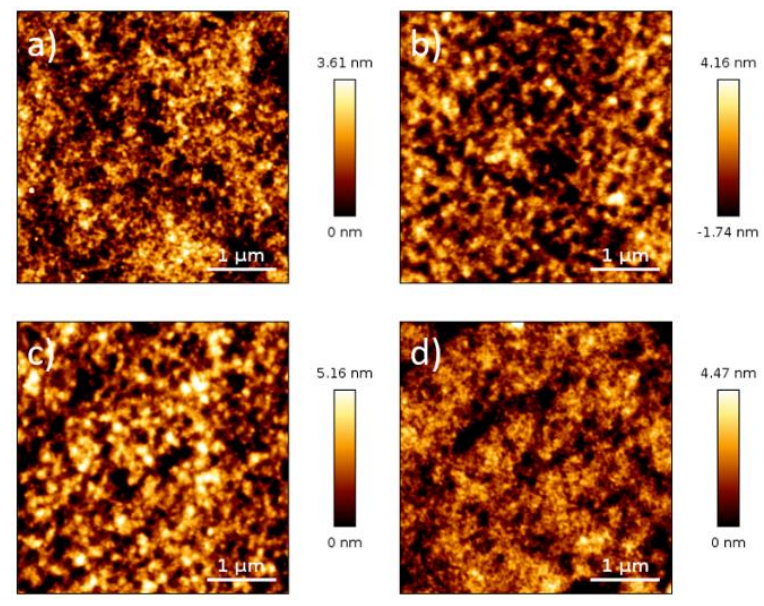

Figure 4. AFM images for a) PTB7-Th:PTbPDI, b) PTB7-Th:PTTbPDI, c) PTB7-Th:PTTTbPDI and d) PTB7Th:PBDTbPDI all-polymer solar cells.

We then turned back to the projected benefits of bis-PDI acceptor polymers in terms of straightforward synthesis and photostability. The ease of synthesis of the four polymers was analyzed by determining their synthetic complexity (SC), calculated according to the method proposed by Po and co-workers (see Supplementary data).[24] For PTbPDI, an SC of 29 was obtained, whereas the well-known ITIC molecular acceptor has a much higher SC of 57 (Table S6). The bis-PDI polymers are hence much more attractive for future applications in terms of material cost.

Stability under the combined influence of light and oxygen is a basic test criterion for solar cell materials. We hence studied the behavior of the bis-PDI polymers, spin-coated on glass substrates, under controlled light exposure (194 $\pm 6 \mathrm{~mW} \mathrm{~cm}^{-2}$ from a $447 \mathrm{~nm}$ LED array source, see SI) in air. The stability was monitored by the changes in the UV-Vis optical absorption spectra, as shown in Figures $5 \mathrm{a}$ and $5 \mathrm{~b}$ for spin-coated films of PTbPDI and PBDTbPDI (spectra for PTTbPDI and PTTTbPDI in Figure S26). The acceptor polymers all show a band in the region corresponding to the absorption of the bis-PDI building block (shown for comparison in Figure 5b), however with an additional feature at the low-energy side that is ascribed to intramolecular charge transfer between the 'push' and 'pull' type building blocks. Under the degradation 
treatment, this long-wavelength feature is bleached first while the characteristic bis-PDI band remains. This observation points to a gradual disruption in the polymer chain, whereas the bis-PDI segments are staying quite untouched. For the TT, TTT to BDT cases, one also observes a correlated bleaching of a higher energy band corresponding well in position with those of the electron-rich monomers (marked in Figures $5 b$ and S26). The spectrum of the BDT linker is shown for comparison in Figure $5 b$ while for oligothiophenes this is found in literature.[43]
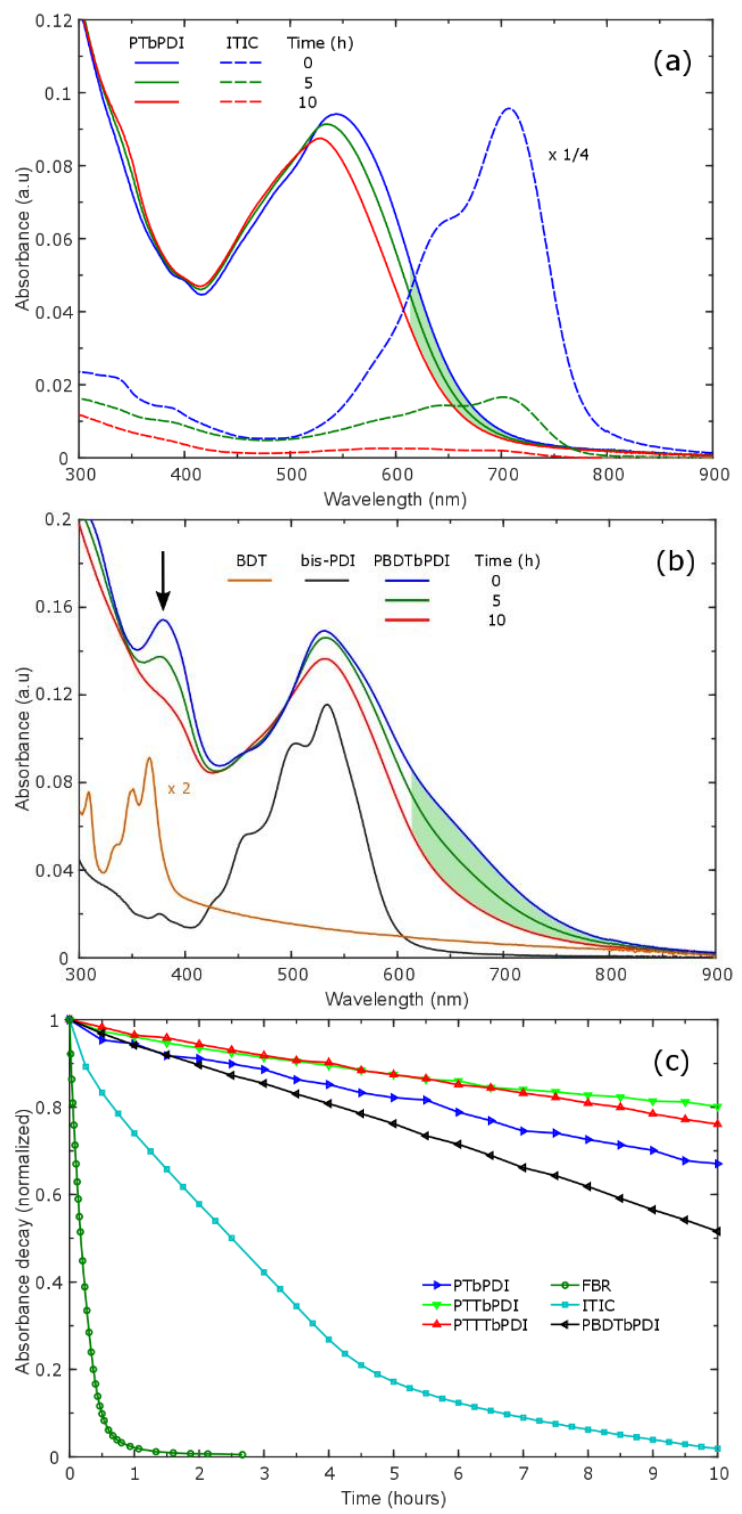

Figure 5. UV-Vis absorption spectra of spin-coated films of a) PTbPDI and ITIC and b) PBDTbPDI before and after different times of photodegradation in air. In b), the spectra of the bis-PDI and BDT molecules are included for comparison and the arrow marks the region of bleaching attributed to transitions of the BDT linking unit. Pane $\mathrm{c}$ ) shows the photodegradation curves of the bis-PDI polymers compared to those 
of the NFA molecules FBR and ITIC. In a) and b), the green-shaded area illustrates the change in integrated absorbance.

The bleaching of the characteristic long-wavelength feature is shown in Figure $5 c$ over a period of $10 \mathrm{~h}$. As the quantity for monitoring of the degradation, we have taken the absorbance integrated in the range most sensitive to the treatment (marked by the green area), and beyond the more resilient bis-PDI like band (> $615 \mathrm{~nm}$ ) (detailed in SI), normalized to the initial value. This way the monitoring is targeting most sensitively the disappearance of the long-wavelength feature characteristic for the pristine polymers. Even in the harsh conditions of full air exposure and intense short-wavelength illumination, the degradation of the acceptor polymers is slow. Indeed, the half-value time is more than 8 hours for PBDTbPDI, and it is even over 15 hours (linearly extrapolated) for the polymers with thiophene linkers. Among the latter, PTbPDI seems to be somewhat more vulnerable than PTTbPDI and PTTTbPDI.

For comparison, we also include here the results of the same degradation procedure applied to two relevant small molecule acceptors for fullerene-free bulk heterojunction organic solar cells (chemical structures in Figure S28). FBR is one of the pioneer NFAs, while ITIC is a top-ranking acceptor that affords close-to-record solar cell efficiencies in combination with well-chosen donor polymers.[10, 44] FBR and ITIC possess a prominent long-wavelength band with a maximum at 508 and $708 \mathrm{~nm}$, respectively (UV-Vis spectra of spin-coated films in Figure 5a and S26). The absorbance at this maximum, normalized to the initial value, is plotted for these materials in Figure $5 c$ as the relevant observable for degradation. This band ultimately completely bleaches under the applied treatment, unlike the absorption spectra of our PDI-based polymers in which the bis-PDI-related feature was persisting. FBR shows a very fast degradation, remaining with only half of the absorbance within 15 minutes. Also for ITIC a significantly lower photostability than for the bis-PDI-based polymers is found, with half of the initial absorbance reached within 2.5-5 hours (the quite large variations on this characteristic time was found to correlate with aggregation effects in the ITIC film: see SI, Figure S27b).

\section{Conclusions}

Four acceptor-type polymers based on the bis(perylene diimide) electron-deficient monomer (with little synthetic complexity) were successfully synthesized. When using these materials in polymer solar cells in combination with PTB7-Th as the donor polymer, average power conversion efficiencies ranging from 3.2 to $4.7 \%$ were achieved. Within the polymer series, PTbPDI afforded the highest efficiency, with a maximum of $4.7 \%$. To the best of our knowledge, this is the highest efficiency achieved so far for a bis-PDI acceptor polymer. The photostability in air of the bis-PDI based polymers, in particular the ones with the 
oligothiophene linkers, was found to be significantly higher than for two standard non-fullerene acceptors, as monitored by bleaching of their relevant optical absorption features. These results show that the bis(perylene diimide) moiety is a viable electron-deficient monomer to be used in acceptor materials for all-polymer solar cells. Higher power conversion efficiencies might be achieved by testing other donor polymers, preferably with a lower gap and an absorption profile even more complementary to the acceptor polymers.

\section{Acknowledgements}

This work is supported by the Research Foundation - Flanders (FWO Vlaanderen) (project G.0B67.15N and SB doctoral fellowship I. Sudakov). J. Kesters and P. Verstappen are postdoctoral fellows of the FWO Vlaanderen. The calculations were performed on the computers of the Consortium des Équipements de Calcul Intensif (CECl, http://www.ceci-hpc.be), including those of the Technological Platform of HighPerformance Computing, for which we gratefully acknowledge the financial support of the FNRS-FRFC (Conventions No. 2.4.617.07.F and 2.5020.11) and the University of Namur. The authors also like to thank H. Penxten for the CV measurements.

\section{Appendix A. Supplementary material}

Supplementary data associated with this article can be found in the online version at doi:10.1016/j.solmat....

\section{References}

[1] K.A. Mazzio, C.K. Luscombe, The future of organic photovoltaics, Chem. Soc. Rev., 44 (2015) 78-90. https://doi.org/10.1039/c4cs00227j.

[2] S. Holliday, Y.L. Li, C.K. Luscombe, Recent advances in high performance donor-acceptor polymers for $\begin{array}{llllll}\text { organic photovoltaics, } & \text { Prog. } & \text { Polym. } & \text { Sci., } & 70 & \text { (2017) }\end{array}$ https://doi.org/10.1016/j.progpolymsci.2017.03.003.

[3] J.B. Zhao, Y.K. Li, G.F. Yang, K. Jiang, H.R. Lin, H. Ade, W. Ma, H. Yan, Efficient organic solar cells processed from hydrocarbon solvents, Nat. Energy, 1 (2016) 15027. https://doi.org/10.1038/Nenergy.2015.27.

[4] D. Deng, Y. Zhang, J. Zhang, Z. Wang, L. Zhu, J. Fang, B. Xia, Z. Wang, K. Lu, W. Ma, Z. Wei, Fluorinationenabled optimal morphology leads to over $11 \%$ efficiency for inverted small-molecule organic solar cells, Nat. Commun, 7 (2016) 13740. https://doi.org/10.1038/ncomms13740.

[5] Z. Zheng, S. Zhang, J. Zhang, Y. Qin, W. Li, R. Yu, Z. Wei, J. Hou, Over 11\% Efficiency in Tandem Polymer Solar Cells Featured by a Low-Band-Gap Polymer with Fine-Tuned Properties, Adv. Mater., 28 (2016) 51335138. https://doi.org/10.1002/adma.201600373.

[6] H. Zhou, Y. Zhang, C.K. Mai, S.D. Collins, G.C. Bazan, T.Q. Nguyen, A.J. Heeger, Polymer homo-tandem solar cells with best efficiency of 11.3\%, Adv. Mater., 27 (2015) 1767-1773. https://doi.org/10.1002/adma.201404220. 
[7] S. Lizin, S. Van Passel, E. De Schepper, W. Maes, L. Lutsen, J. Manca, D. Vanderzande, Life cycle analyses of organic photovoltaics: a review, Energy Environ. Sci., 6 (2013) 3136-3149. https://doi.org/10.1039/c3ee42653j.

[8] I. Cardinaletti, J. Kesters, S. Bertho, B. Conings, F. Piersimoni, J. D'Haen, L. Lutsen, M. Nesladek, B. Van Mele, G. Van Assche, K. Vandewal, A. Salleo, D. Vanderzande, W. Maes, J.V. Manca, Toward bulk heterojunction polymer solar cells with thermally stable active layer morphology, J. Photonics Energy, 4 (2014) 040997. https://doi.org/10.1117/1.jpe.4.040997.

[9] D. Baran, R.S. Ashraf, D.A. Hanifi, M. Abdelsamie, N. Gasparini, J.A. Rohr, S. Holliday, A. Wadsworth, S. Lockett, M. Neophytou, C.J. Emmott, J. Nelson, C.J. Brabec, A. Amassian, A. Salleo, T. Kirchartz, J.R. Durrant, I. McCulloch, Reducing the efficiency-stability-cost gap of organic photovoltaics with highly efficient and stable small molecule acceptor ternary solar cells, Nat. Mater., 16 (2017) 363-369. https://doi.org/10.1038/nmat4797.

[10] W. Zhao, D. Qian, S. Zhang, S. Li, O. Inganas, F. Gao, J. Hou, Fullerene-Free Polymer Solar Cells with over 11\% Efficiency and Excellent Thermal Stability, Adv. Mater., 28 (2016) 4734-4739. https://doi.org/10.1002/adma.201600281.

[11] P. Cheng, H. Bai, N.K. Zawacka, T.R. Andersen, W. Liu, E. Bundgaard, M. Jorgensen, H. Chen, F.C. Krebs, X. Zhan, Roll-Coated Fabrication of Fullerene-Free Organic Solar Cells with Improved Stability, Adv. Sci., 2 (2015) 1500096. https://doi.org/10.1002/advs.201500096.

[12] Y. Lin, X. Zhan, Non-fullerene acceptors for organic photovoltaics: an emerging horizon, Mater. Horizons, 1 (2014) 470-488. https://doi.org/10.1039/c4mh00042k.

[13] W. Zhao, S. Li, H. Yao, S. Zhang, Y. Zhang, B. Yang, J. Hou, Molecular Optimization Enables over 13\% Efficiency in Organic Solar Cells, J. Am. Chem. Soc, 139 (2017) 7148-7151. https://doi.org/10.1021/jacs.7b02677.

[14] J. Hou, O. Inganäs, R.H. Friend, F. Gao, Organic solar cells based on non-fullerene acceptors, Nat. Mater, 17 (2018) 119-128. https://doi.org/10.1038/nmat5063.

[15] S. Li, L. Ye, W. Zhao, H. Yan, B. Yang, D. Liu, W. Li, H. Ade, J. Hou, A Wide Band Gap Polymer with a Deep Highest Occupied Molecular Orbital Level Enables 14.2\% Efficiency in Polymer Solar Cells, J. Am. Chem. Soc, (2018) 7159-7167. https://doi.org/10.1021/jacs.8b02695.

[16] L. Meng, Y. Zhang, X. Wan, C. Li, X. Zhang, Y. Wang, X. Ke, Z. Xiao, L. Ding, R. Xia, H.L. Yip, Y. Cao, Y. Chen, Organic and solution-processed tandem solar cells with $17.3 \%$ efficiency, Science, (2018) 10941098. https://doi.org/10.1126/science.aat2612.

[17] C. Yan, S. Barlow, Z. Wang, H. Yan, A.K.Y. Jen, S.R. Marder, X. Zhan, Non-fullerene acceptors for organic solar cells, Nat. Rev. Mater., 3 (2018) 18003. https://doi.org/10.1038/natrevmats.2018.3.

[18] S. Li, Z. Zhang, M. Shi, C.Z. Li, H. Chen, Molecular electron acceptors for efficient fullerene-free organic solar cells, Phys. Chem. Chem. Phys., 19 (2017) 3440-3458. https://doi.org/10.1039/c6cp07465k.

[19] Z. Li, X. Xu, W. Zhang, X. Meng, Z. Genene, W. Ma, W. Mammo, A. Yartsev, M.R. Andersson, R.A.J. Janssen, E. Wang, 9.0\% power conversion efficiency from ternary all-polymer solar cells, Energy Environ. Sci., 10 (2017) 2212-2221. https://doi.org/10.1039/c7ee01858d.

[20] Z. Li, L. Ying, P. Zhu, W. Zhong, N. Li, F. Liu, F. Huang, Y. Cao, A generic green solvent concept boosting the power conversion efficiency of all-polymer solar cells to 11\%, Energy Environ. Sci., (2018). https://doi.org/10.1039/c8ee02863j.

[21] B. Fan, L. Ying, P. Zhu, F. Pan, F. Liu, J. Chen, F. Huang, Y. Cao, All-Polymer Solar Cells Based on a Conjugated Polymer Containing Siloxane-Functionalized Side Chains with Efficiency over 10\%, Adv. Mater., 29 (2017) 17033906. https://doi.org/10.1002/adma.201703906.

[22] Y. Zhang, Y. Xu, M.J. Ford, F. Li, J. Sun, X. Ling, Y. Wang, J. Gu, J. Yuan, W. Ma, Thermally Stable AllPolymer Solar Cells with High Tolerance on Blend Ratios, Adv. Energy Mater., (2018) 1800029. https://doi.org/10.1002/aenm.201800029. 
[23] X. Liu, C. Zhang, C. Duan, M. Li, Z. Hu, J. Wang, F. Liu, N. Li, C.J. Brabec, R.A.J. Janssen, G.C. Bazan, F. Huang, Y. Cao, Morphology Optimization via Side Chain Engineering Enables All-Polymer Solar Cells with Excellent Fill Factor and Stability, J. Am. Chem. Soc, 140 (2018) 8934-8943. https://doi.org/10.1021/jacs.8b05038.

[24] R. Po, G. Bianchi, C. Carbonera, A. Pellegrino, "All That Glisters Is Not Gold": An Analysis of the Synthetic Complexity of Efficient Polymer Donors for Polymer Solar Cells, Macromolecules, 48 (2015) 453461. https://doi.org/10.1021/ma501894w.

[25] C. Li, H. Wonneberger, Perylene imides for organic photovoltaics: yesterday, today, and tomorrow, Adv. Mater., 24 (2012) 613-636. https://doi.org/10.1002/adma.201104447.

[26] L. Hu, Y. Liu, L. Mao, S.X. Xiong, L.L. Sun, N. Zhao, F. Qin, Y.Y. Jiang, Y.H. Zhou, Chemical reaction between an ITIC electron acceptor and an amine-containing interfacial layer in non-fullerene solar cells, J. Mater. Chem. A, 6 (2018) 2273-2278. https://doi.org/10.1039/c7ta10306a.

[27] F. Fernández-Lázaro, N. Zink-Lorre, Á. Sastre-Santos, Perylenediimides as non-fullerene acceptors in bulk-heterojunction solar cells (BHJSCs), J. Mater. Chem. A, 4 (2016) 9336-9346. https://doi.org/10.1039/c6ta02045c.

[28] C.-H. Wu, C.-C. Chueh, Y.-Y. Xi, H.-L. Zhong, G.-P. Gao, Z.-H. Wang, L.D. Pozzo, T.-C. Wen, A.K.Y. Jen, Influence of Molecular Geometry of Perylene Diimide Dimers and Polymers on Bulk Heterojunction Morphology Toward High-Performance Nonfullerene Polymer Solar Cells, Adv. Funct. Mater, 25 (2015) 5326-5332. https://doi.org/10.1002/adfm.201501971.

[29] F. Yang, C. Li, G.-T. Feng, X.-D. Jiang, A.-D. Zhang, W.-W. Li, Bisperylene bisimide based conjugated polymer as electron acceptor for polymer-polymer solar cells, Chin. J. Polym. Sci., 35 (2016) 239-248. https://doi.org/10.1007/s10118-017-1870-4.

[30] Y. Hu, S. Chen, L. Zhang, Y. Zhang, Z. Yuan, X. Zhao, Y. Chen, Facile Approach to Perylenemonoimide with Short Side Chains for Nonfullerene Solar Cells, J. Org. Chem., 82 (2017) 5926-5931. https://doi.org/10.1021/acs.joc.7b00272.

[31] Y. Yu, F. Yang, Y. Ji, Y. Wu, A. Zhang, C. Li, W. Li, A perylene bisimide derivative with a LUMO level of $-4.56 \mathrm{eV}$ for non-fullerene solar cells, J. Mater. Chem. C, 4 (2016) 4134-4137. https://doi.org/10.1039/c6tc01045h.

[32] W. Jiang, L. Ye, X. Li, C. Xiao, F. Tan, W. Zhao, J. Hou, Z. Wang, Bay-linked perylene bisimides as promising non-fullerene acceptors for organic solar cells, Chem. Commun., 50 (2014) 1024-1026. https://doi.org/10.1039/c3cc47204c.

[33] Y. Yang, Y. Wang, Y. Xie, T. Xiong, Z. Yuan, Y. Zhang, S. Qian, Y. Xiao, Fused perylenebisimide-carbazole: new ladder chromophores with enhanced third-order nonlinear optical activities, Chem. Commun., 47 (2011) 10749-10751. https://doi.org/10.1039/c1cc14071j.

[34] X. Liu, Y.J. Kim, M.-J. Kim, C.E. Park, Y.-H. Kim, A unique concept of copolymer composed of main chain donor and side chain acceptor for promising bulk heterojunction solar cells, Synth. Met., 205 (2015) 195-200. https://doi.org/10.1016/j.synthmet.2015.04.012.

[35] E. Kozma, D. Kotowski, F. Bertini, S. Luzzati, M. Catellani, Synthesis of donor-acceptor poly(perylene diimide-altoligothiophene) copolymers as n-type materials for polymeric solar cells, Polymer, 51 (2010) 2264-2270. https://doi.org/10.1016/j.polymer.2010.03.040.

[36] C. Cabanetos, A. El Labban, J.A. Bartelt, J.D. Douglas, W.R. Mateker, J.M. Fréchet, M.D. McGehee, P.M. Beaujuge, Linear side chains in benzo[1,2-b:4,5-b']dithiophene-thieno[3,4-c]pyrrole-4,6-dione polymers direct self-assembly and solar cell performance, J. Am. Chem. Soc., 135 (2013) 4656-4659. https://doi.org/10.1021/ja400365b.

[37] J. Hou, M.-H. Park, S. Zhang, Y. Yao, L.-M. Chen, J.-H. Li, Y. Yang, Bandgap and Molecular Energy Level Control of Conjugated Polymer Photovoltaic Materials Based on Benzo[1,2-b:4,5-b']dithiophene, Macromolecules, 41 (2008) 6012-6018. https://doi.org/10.1021/ma800820r. 
[38] Y. He, Y. Zhou, G. Zhao, J. Min, X. Guo, B. Zhang, M. Zhang, J. Zhang, Y. Li, F. Zhang, O. Inganäs, Poly(4,8bis(2-ethylhexyloxy)benzo[1,2-b:4,5-b']dithiophene vinylene): Synthesis, optical and photovoltaic properties, J. Polym. Sci. A, 48 (2010) 1822-1829. https://doi.org/10.1002/pola.23952.

[39] G.W.T. M. J. Frisch, H. B. Schlegel, G. E. Scuseria, M. A. Robb, J. R. Cheeseman, G. Scalmani, V. Barone, B. Mennucci, G. A. Petersson, H. Nakatsuji, M. Caricato, X. Li, H. P. Hratchian, A. F. Izmaylov, J. Bloino, G. Zheng, J. L. Sonnenberg, M. Hada, M. Ehara, K. Toyota, R. Fukuda, J. Hasegawa, M. Ishida, T. Nakajima, Y. Honda, O. Kitao, H. Nakai, T. Vreven, J. A. Montgomery, Jr., J. E. Peralta, F. Ogliaro, M. Bearpark, J. J. Heyd, E. Brothers, K. N. Kudin, V. N. Staroverov, R. Kobayashi, J. Normand, K. Raghavachari, A. Rendell, J. C. Burant, S. S. Iyengar, J. Tomasi, M. Cossi, N. Rega, J. M. Millam, M. Klene, J. E. Knox, J. B. Cross, V. Bakken, C. Adamo, J. Jaramillo, R. Gomperts, R. E. Stratmann, O. Yazyev, A. J. Austin, R. Cammi, C. Pomelli, J. W. Ochterski, R. L. Martin, K. Morokuma, V. G. Zakrzewski, G. A. Voth, P. Salvador, J. J. Dannenberg, S. Dapprich, A. D. Daniels, Ö. Farkas, J. B. Foresman, J. V. Ortiz, J. Cioslowski, D. J. Fox, Gaussian-09 Revision D.01, Gaussian Inc., Wallingford CT, 2009.

[40] Y. Zhao, D.G. Truhlar, The M06 suite of density functionals for main group thermochemistry, thermochemical kinetics, noncovalent interactions, excited states, and transition elements: two new functionals and systematic testing of four M06-class functionals and 12 other functionals, Theor. Chem. Acc., 120 (2007) 215-241. https://doi.org/10.1007/s00214-007-0310-x.

[41] N. Zhou, A.S. Dudnik, T.I. Li, E.F. Manley, T.J. Aldrich, P. Guo, H.C. Liao, Z. Chen, L.X. Chen, R.P. Chang, A. Facchetti, M. Olvera de la Cruz, T.J. Marks, All-Polymer Solar Cell Performance Optimized via Systematic Molecular Weight Tuning of Both Donor and Acceptor Polymers, J. Am. Chem. Soc., 138 (2016) 1240-1251. https://doi.org/10.1021/jacs.5b10735.

[42] N.K. Elumalai, A. Uddin, Open circuit voltage of organic solar cells: an in-depth review, Energy Environ. Sci., 9 (2016) 391-410. https://doi.org/10.1039/c5ee02871j.

[43] J.H. Choi, D.W. Cho, H.J. Park, S.-H. Jin, S. Jung, M. Yi, C.K. Song, U.C. Yoon, Synthesis and characterization of a series of bis(dimethyl-n-octylsilyl)oligothiophenes for organic thin film transistor applications, Synth. Met., 159 (2009) 1589-1596. https://doi.org/10.1016/j.synthmet.2009.04.022.

[44] S. Holliday, R.S. Ashraf, C.B. Nielsen, M. Kirkus, J.A. Rohr, C.H. Tan, E. Collado-Fregoso, A.C. Knall, J.R. Durrant, J. Nelson, I. McCulloch, A rhodanine flanked nonfullerene acceptor for solution-processed organic photovoltaics, J. Am. Chem. Soc., 137 (2015) 898-904. https://doi.org/10.1021/ja5110602. 